\title{
P53 suppresses expression of the 14-3-3gamma oncogene
}

Vijayababu M Radhakrishnan ${ }^{1,2}$, Charles W Putnam ${ }^{1,3}$, Wenqing Qi, ${ }^{1,4}$ and Jesse D Martinez ${ }^{1 *}$

\begin{abstract}
Background: 14-3-3 proteins are a family of highly conserved proteins that are involved in a wide range of cellular processes. Recent evidence indicates that some of these proteins have oncogenic activity and that they may promote tumorigenesis. We previously showed that one of the 14-3-3 family members, 14-3-3gamma, is over expressed in human lung cancers and that it can induce transformation of rodent cells in vitro.

Methods: qRTPCR and Western blot analysis were performed to examine 14-3-3gamma expression in non-small cell lung cancers (NSCLC). Gene copy number was analyzed by qPCR. P53 mutations were detected by direct sequencing and also by western blot. CHIP and yeast one hybrid assays were used to detect p53 binding to 14-33gamma promoter.

Results: Quantitative rtPCR results showed that the expression level of 14-3-3gamma was elevated in the majority of NSCLC that we examined which was also consistent with protein expression. Further analysis of the expression pattern of 14-3-3gamma in lung tumors showed a correlation with p53 mutations suggesting that p53 might suppress 14-3-3 gamma expression. Analysis of the gamma promoter sequence revealed the presence of a p53 consensus binding motif and in vitro assays demonstrated that wild-type p53 bound to this motif when activated by ionizing radiation. Deletion of the p53 binding motif eliminated p53's ability to suppress 14-3-3gamma expression.
\end{abstract}

Conclusion: Increased expression of 14-3-3gamma in lung cancer coincides with loss of functional p53. Hence, we propose that 14-3-3gamma's oncogenic activities cooperate with loss of p53 to promote lung tumorigenesis.

Keywords: Lung cancer, 14-3-3, p53 mutations, Gene Copy, Transcription Regulation

\section{Background}

14-3-3 proteins are present in all eukaryotic organisms that have been examined and are highly conserved between species. The number of proteins in the 14-3-3 family varies with species. However, in mammals, seven isoforms have been identified named as $\beta, \gamma, \varepsilon, \sigma, \zeta, \theta$ and $\eta$, and they function by binding other proteins predominantly through phosphorylated serine residues $[1,2]$. These proteins are highly conserved and are involved in the regulation of a variety of key physiological pathways such as cell cycle progression [3] apoptosis [4] and mitogenic signaling [5]. Binding target proteins enable 14-3-3 family members to regulate the activity of

\footnotetext{
* Correspondence: jmartinez@azcc.arizona.edu

'Arizona Cancer Center, Department of Cellular \& Molecular Medicine,

University of Arizona, Tucson, Arizona 85724, USA

Full list of author information is available at the end of the article
}

enzymes, control subcellular localization of their targets, or act as scaffolds that promote protein-protein interactions.

14-3-3 proteins were identified as abundant proteins in the brain and were first described to activate neurotransmitter synthesis [6]. Subsequently, they were implicated in a variety of neurological conditions [7] suggesting that they functioned primarily in the brain. However, 14-3-3 protein family members are widely expressed in mammalian tissues and recent evidence suggests that these proteins may also play a role in the development of human cancers. Examination of 14-3-3 protein levels in human tumors including lung [8], prostate [9], breast [10], oral [11], ovarian [12] and pancreatic cancers $[13,14]$ indicate that $14-3-3$ protein expression becomes aberrant during tumorigenesis.

\section{Biomed Central}


However, it is unclear if or how these proteins contribute to tumorigenesis.

Of the 14-3-3 proteins linked to cancer, the best studied is $14-3-3 \sigma$, which is a transcriptional target of the p53 tumor suppressor. Activation of p53 by DNA damage leads to induction of $14-3-3 \sigma$ and G2 arrest [3]. Loss of 14-3-3 $\sigma$ also results in defective DNA damage repair [15] and promotes tumorigenesis in breast epithelia [16]. Moreover, down regulation of 14-3-3 $\sigma$ enables primary human epithelial cells to grow indefinitely [17]. Collectively these findings suggest that 14-3-30 may function as a tumor suppressor and confirm that 14-3-3 gene expression can be regulated by $\mathrm{p} 53$.

The role of 14-3-3 $\gamma$ isoform in cancer is less well understood. However, Jin et al. [18] have shown that 14$3-3 \gamma$ can inhibit transcriptional activity of p53 and we have previously shown that the $14-3-3 \gamma$ protein is overexpressed in lung cancers and can promote polyploidy

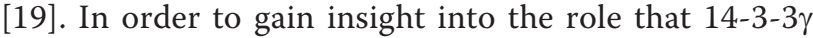
may have in lung tumorigenesis we examined their expression and the co-occurrence of p53 mutations in lung tumor specimens. We found evidence suggestive of a functional interaction between 14-3-3 $\gamma$ and $\mathrm{p} 53$.

\section{Methods}

Frozen human lung tumor specimens and non malignant tissues were obtained from Cooperative Human Tissue Network, Vanderbilt University Medical Center (Nashville, TN). 80 samples were selected based on the tumor type and percentage of tumor cell content (> $70 \%)$ and also 21 normal tissues were selected. These studies were evaluated by the University of Arizona Human Subjects Protection Program and judged to be exempt as the specimens are de-identified. The human lung cancer cells, A549, H358 and H322 cells were obtained from American Type Culture Collection (ATCC), USA. The human colorectal cancer cell lines p $53^{+/+}$and $\mathrm{p} 53^{-/-}$HCT 116 were provided by Dr. Bert Vogelstein (The Johns Hopkins University). Anti-p53 and Anti-14-3-3 $\gamma$ antibodies were obtained from Santa Cruz (Santa Cruz, CA). Antibody to $\beta$-actin was purchased from Sigma, St Louis, MO. PCR kits were obtained from Invitrogen, USA. First strand cDNA synthesis kit was obtained from Fermentas, USA.

\section{Real-Time PCR quantitation of mRNA expression for 14-3-} $3 \gamma$

The mRNA expression level was determined by quantitative reverse transcription-PCR on total RNA, using the ABI PRISM 7700 Sequence Detection System. The RNA was isolated using TRIZOL (Invitrogen, USA) and treated with DNase to avoid amplification of genomic DNA. cDNA was synthesized from $500 \mathrm{ng}$ of total RNA and it was used for PCR. PCR was carried out in $50 \mu \mathrm{l}$ reaction mixture containing $25 \mu \mathrm{l}$ of SYBR Green qPCR SuperMix-UDG, $1.5 \mu \mathrm{l}$ of $10 \mu \mathrm{M}$ each forward and reverse primer, $2 \mu \mathrm{l}$ of cDNA and water to $50 \mu \mathrm{l}$. Thermal cycling conditions were carried at $50^{\circ} \mathrm{C}$ for $2 \mathrm{~min}, 95^{\circ} \mathrm{C}$ for $2 \mathrm{~min}, 40$ cycles of $95^{\circ} \mathrm{C}$ for $30 \mathrm{sec}$ and $58^{\circ} \mathrm{C}$ for 30 sec using the ABI PRISM 7700 Sequence Detection System. The primers for 14-3-3 $\gamma$ and GAPDH transcript are listed in Table 1. All experiments were repeated three times. Data are expressed as $\Delta \mathrm{Ct}$ values $[\Delta \mathrm{Ct}=\mathrm{Ct}$

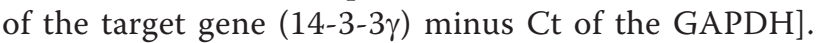
To calculate number of fold changes compared with normal tissues, $2^{-\Delta \Delta C t}$ equation was used; all statistics were performed with $\Delta \mathrm{Ct}$ values.

\section{Quantitative-PCR for relative gene quantity of 14-3-3 $\gamma$}

Normal and tumor specimens were embedded in OCT and sections were prepared using a MICROM GmbH cryostat (Waldorf, Germany). The sections were fixed in $75 \%$ ethanol and stained in $1.5 \%$ eosin for $30 \mathrm{sec}$. Then they were washed in 95\% ethanol, dehydrated in 100\% high-grade ethanol, incubated with Xylene, air dried, and subjected to laser capture microdissection (LCM) using the Veritas Microdissection instrument (Arcturus Engineering, CA) and CapSure LCM Caps (Arcturus Engineering, CA). DNA was extracted using the Picopure DNA extraction kit (Arcturus Engineering, CA). Q-PCR was performed using the ABI PRISM 7700 sequence Detection System (Applied Biosystems, USA) by TaqMan based technique. The sequences of the genomic primers and probes used for Q-PCR are listed in Table 2. The primers were tested to ensure amplification of single discrete bands. Relative quantification was performed using the comparative CT $(\Delta \mathrm{CT})$ method. To normalize the Q-PCR data against aneuploidy that commonly occurs in NSCLCs [20], the Ct values of the 143-3 $\gamma$ gene are normalized with phosphoinositide-3kinase regulatory subunit 1 (PI3KR1) gene, which is unaltered in NSCLCs [21]. Each real-time qPCR reaction mixture contained $10 \mu \mathrm{l}$ of DNA sample (obtained from Picopure DNA extraction kit), $12.5 \mu$ l of Platinum Quantitative PCR SuperMix-UDG (Invitrogen, USA), $0.75 \mu \mathrm{l}$ of $10 \mu \mathrm{M}$ gene-specific forward and reverse primers, $0.25 \mu \mathrm{l}$ of $10 \mu \mathrm{M}$ fluorescent probe and water to $25 \mu \mathrm{l}$. Thermal cycling conditions were carried at $50^{\circ} \mathrm{C}$ for $2 \mathrm{~min}, 95^{\circ} \mathrm{C}$ for $2 \mathrm{~min}, 40$ cycles of $95^{\circ} \mathrm{C}$ for $30 \mathrm{sec}$ and $58^{\circ} \mathrm{C}$ for 30 sec using the ABI PRISM 7700 Sequence Detection System. Samples were run on a $2 \%$

Table 1 List of primer sequences used for mRNA expression of 14-3-3 $\gamma$ and GAPDH

\begin{tabular}{lcc}
\hline & Forward $\left(\mathbf{5}^{\prime}>\mathbf{3}^{\prime}\right)$ & Reverse $\left(\mathbf{5}^{\prime} \mathbf{>}^{\prime} \mathbf{3}^{\prime}\right)$ \\
\hline $\mathbf{1 4 - 3 - 3} \boldsymbol{\gamma}$ & CTGAATGAGCCACTGTCGAA & GCACGGACCATCTCAATCTT \\
GAPDH & AGGGCCCTGACAACTCTITT & AGGGGTCTACATGGCAACTG \\
\hline
\end{tabular}


Table 2 List of primer sequences used for amplification of 14-3-3 $\gamma$ and PI3KR1 genes by Qpcr

\begin{tabular}{|c|c|c|c|}
\hline & Forward $\left(5^{\prime}>3^{\prime}\right)$ & Reverse $\left(5^{\prime}>3^{\prime}\right)$ & Probe $\left(5^{\prime}>3^{\prime}\right)$ \\
\hline $14-3-3 \gamma$ & CCTCAGCTGCTCGCTCTG & CGGAGAAGGAGGAGGACACT & $\begin{array}{l}\text { 6-FAM- } \\
\text { CGGTCCTCTCCGGCACTTGGGC-TAMRA }\end{array}$ \\
\hline PI3KR1 & $\begin{array}{l}\text { AAGTCTTAAGTTTGGGTTG } \\
\text { AGTCG }\end{array}$ & $\begin{array}{l}\text { TAATGATTGACCAAGCTITIA } \\
\text { TGC }\end{array}$ & $\begin{array}{l}\text { 6-FAM- } \\
\text { TAATGATTGACCAAGCTITTATGC-TAMRA }\end{array}$ \\
\hline
\end{tabular}

agarose gel to ensure that only a single amplicon was produced.

\section{RT-PCR and Direct sequencing for p53 mutations}

For the mutational analysis of p53 gene exons 5-9, RTPCR analysis and direct sequencing were performed. cDNA from tumor samples was synthesized from 500 ng of total RNA and then PCR was performed using following primers, forward 5'-GCC AAG TCT GTG ACT TGC ACG-3' and reverse 5'-AGA GGA GCT GGT GTT GTT GG-3'. The cycling conditions used for these PCRs were as follows: $94^{\circ} \mathrm{C}$ for $5 \mathrm{~min}, 30$ cycles of $94^{\circ} \mathrm{C}$ for $30 \mathrm{sec}, 55^{\circ} \mathrm{C}$ for $45 \mathrm{sec}$ and $72^{\circ} \mathrm{C}$ for $1 \mathrm{~min}$, with a final extension step of $72^{\circ} \mathrm{C}$ for $10 \mathrm{~min}$. The PCR products were gel purified using gel purification kit (Qiagen, USA). Purified DNA samples were sequenced at the University of Arizona Genetics Core facility.

\section{Western blot for expression of $14-3-3 \gamma$, p53 and $\beta$-actin proteins}

The protein lysates from either frozen sections or cell lines were collected using ice-cold RIPA buffer containing $150 \mathrm{mM} \mathrm{NaCl}, 50 \mathrm{mM}$ Tris, $1 \mathrm{mM}$ EDTA, 1\% NP40, $0.5 \%$ sodium deoxycholate, $0.1 \%$ SDS, $\mathrm{pH} 7.4$ and 2 $\mu \mathrm{l} / \mathrm{ml}$ Protease inhibitor cocktail (Sigma, USA). Protein concentrations were determined using the BioRad protein assay kit (Biorad, USA) and $50 \mu \mathrm{g}$ protein was separated by $12 \%$ SDS-PAGE gel. The proteins were then transferred onto a nitrocellulose membrane (Millipore, USA) and then blocking was carried out by incubating with 5\% nonfat milk in TBST buffer. After blocking, the membranes were incubated with primary antibodies raised against human 14-3-3 $\gamma$ (Rabbit polyclonal, Santa Cruz, USA), p53 (DO1, mouse monoclonal, Santa Cruz, USA) and $\beta$-actin for $1 \mathrm{hr}$. Then the membranes were washed three times, incubated with secondary-HRP (Sigma, USA) for $1 \mathrm{hr}$ and then washed with TBST buffer for three times. Blots were developed by Super Signal West Pico detection system (Pierce, Rockford, IL). The membranes were stripped and reprobed with $\beta$-actin monoclonal antibody (Sigma, USA) to confirm equal loading. For frozen tumor specimens, the protein quantification from western blot image was done with the help of Image J program (NIH, MD) and 2-fold above the normal is considered as 14-3-3 $\gamma$ overexpressing tumors.

\section{One Hybrid Assays}

The Grow'n'Glow GFP One-Hybrid System (MoBiTec, USA) was used to test for p53 binding to YWHAG promoter sequences. The prey control plasmid pJG4-5-p53, supplied with the kit, consists of the p53 cDNA fused to the B42 activation domain; expression of the p53 fusion protein is under the control of the GAL1 promoter. The 600 and 1200 bp promoter sequences were subcloned into the GFP reporter vector ("bait" plasmid), pGNG2, which contains the $\mathrm{GFP}_{\mathrm{UV}}$ gene driven by the GAL1, 10 minimal promoter. The 600 and $1200 \mathrm{bp}$ fragments were amplified by PCR from the pGL3 plasmid containing complete 14-3-3 $\gamma$ promoter using the following primers: 600 bp forward primer, 5'-GAGAGCGGCCGC GTCGGTCCTCTCCGGCACTT-3'; 1200 bp forward primer, 5'-GAGAGCGGCCGCATGAACGAGAATATATCAGCGTGACC-3'; reverse primer for both reactions, 5'-GAGAACTAGTCTTCGCGGGGCTGGGTCT3'. A NotI recognition sequence is incorporated into the forward primers; the reverse primer includes a SpeI recognition sequence. The amplification products and the pGNG2 plasmid were cut with NotI and SpeI, and the promoter sequences were ligated into the vector. The two promoter constructs were verified by sequencing. The p53 encoding plasmid was co-transformed with reporter construct into Saccharomyces cerevisiae strain, W303 and successful transformants selected on -ura -trp synthetic medium containing dextrose. Individual colonies were inoculated into - ura -trp SC medium with $2 \%$ dextrose and grown to mid-log phase. The cells were then split in two, washed with sterile water, and resuspended in -ura -trp SC medium containing either $2 \%$ sucrose or $2 \%$ galactose. After six hours, cell density was measured spectrophotometrically $\left(\mathrm{OD}_{600}\right)$ and $200 \mu \mathrm{L}$ aliquots loaded onto a 96-well microplate. The plates were inspected visually under long wave UV light for green fluorescence; the fluorescence was quantified using a plate reader (excitation at $395 \mathrm{~nm}$ and emission at 509 $\mathrm{nm}$ ) (Molecular Devices, USA). The mean values for each promoter construct and carbon source were normalized for cell density and expressed as the ratio of fluorescence in galactose to that in sucrose, the latter set at one fold.

\section{ChIP assays}

We collected A549, H358, H322, HCT116p53 $3^{+/+}$and HCT $116 \mathrm{p} 53^{-/-}$cells for ChIP assay $8 \mathrm{~h}$ after $\gamma$-irradiation. 
ChIP assays were carried out essentially as described [22]. p53 immunoprecipitation was done with $5 \mu \mathrm{g}$ of antibody against p53 (DO1, Santa Cruz, USA), or with Mouse IgG (Santa Cruz, USA) as a negative control. We carried out PCR amplification using primers (forward, 5'-AACCACTGTGGCCAGCCGGTAT-3'; reverse, 5'ACAGGAGGCGCGTCCATTGT-3'), designed to give a product including the p53-binding element. The PCR protocol was 30 cycles of a $45 \mathrm{sec}$ denaturation step at $94^{\circ} \mathrm{C}$, a 1 min annealing step at $58^{\circ} \mathrm{C}$ and a 1 -min extension step at $72^{\circ} \mathrm{C}$. The PCR products were resolved by $1.5 \%$ agarose gel electrophoresis.

\section{Plasmid Constructions}

To create the pGL3-100, pGL3-586, pGL3-830, pGL3840, pGL3-850 and pGL3-1200 14-3-3 $\gamma$ promoter plasmids, the genomic DNA from human foreskin fibroblast cells (HFF1 cells, provided by Dr. Rilo, University of Arizona) was PCR amplified with forward primers hanging KpnI site and reverse primers hanging NheI site, products were digested and gel purified followed by ligation with KpnI/NheI digested pGL3 linearized vector. For PGL3-1200 $\Delta 850-840$ and PGL3-1200 $\Delta 850-830,350$ bp fragment upstream of p53 RE (-830 to -850) was amplified by PCR with forward primer hanging KpnI. 840 and 830 bp downstream from p53RE products were amplified with reverse primers hanging NheI site. These products were ligated together with KpnI/NheI digested PGL3 empty vector followed by transformation. All the promoter reporter constructs were sequenced and confirmed at the University of Arizona sequencing facility.

\section{Transfections and Luciferase Assays}

All of the transfections were done in triplicate in 24-well plates. Approximately, $1 \times 10^{3}$ cells/well were seeded 24 $\mathrm{h}$ before transfection. Plasmids were transfected into cells using Lipofectamine reagent (Life Technologies, Inc.). Luciferase assays were performed using the Dual Luciferase Assay System (Promega, USA) that already contains an internal control detectable simultaneously with the luciferase reporter gene. Each experiment was conducted at least in triplicates. Ad-GFP and Ad-P53-GFP adenoviruses are laboratory stocks. Cells were at $60 \%$ confluence when infected with 10MOI. siRNA duplexes targeting the p53 mRNA was chemically synthesized by Dharmacon Research. Their target sequences are as follows: p53, 5'-CAGTCTACCTCCCGCCATA-3' (p53 siRNA-1) and 5'-GAAGAAACCACTGGATGGA-3' (p53 siRNA-2). The control siRNAs is as follows: 5'GGCTACGTCCAGGAGCGCACC-3'.

\section{Statistical analysis}

The statistical analysis was performed by analysis of variance. Only $\Delta \mathrm{Ct}$ values were used for the statistical analysis of gene amplification and mRNA expression. The Dunnett's multiple comparison was used to test the statistical significance between normal tissues and tumor tissues. Pearson correlation was used to correlate fold changes of gene amplification and mRNA followed by Student's t-test.

\section{Results}

Increased levels of 14-3-3 $\gamma$ mRNA in lung tumors

We previously showed using qualitative PCR and immunohistochemistry that transcript levels of six of the seven different 14-3-3 genes were elevated in human non-small cell lung cancer (NSCLC) [8]. We showed that one of these, 14-3-3 $\gamma$, acts as an oncogene in focus formation assays suggesting that it might play a role in lung tumorigenesis [23]. In order to gain insight into the mechanism that resulted in the elevated expression in lung tumors, we first utilized quantitative rtPCR to quantify the relative expression levels and to confirm that the expression of 14-3-3 $\gamma$ was transcriptionally elevated. We examined expression levels of 14-3-3 $\gamma$ in 80 frozen samples of non small cell lung cancer (NSCLC) obtained by surgical resection through the Cooperative Human Tissue Network. Total RNA was extracted from the frozen tissues; cDNA was synthesized, and subjected to quantitative RTPCR. The levels of 14-3-3 $\gamma$ mRNA transcript was significantly increased ( $\mathrm{p}<0.0001)$, by an average of six fold when compared to normal tissues (Figure 1A). Western blotting conducted with a subset of the tumors also confirmed that the levels of 14-3-3 $\gamma$ was elevated (Figure 1B). Hence, the elevated levels of mRNA also resulted in elevated protein levels of 14-3-3 $\gamma$.

\section{Overexpression of $14-3-3 \gamma$ does not result from gene amplification}

Since our initial data indicated that $14-3-3 \gamma$ was overexpressed in lung cancers, we next sought to determine the cause of the elevated mRNA levels. One potential explanation is that the 14-3-3 $\gamma$ gene may be amplified. Consequently, we determined the relative quantity of 14-3-3 $\gamma$ DNA using quantitative PCR and compared the values with values obtained from normal tissue. As can be seen in Figure $1 C$, the quantity of $14-3-3 \gamma$ gene was altered in some of the tumors, but overall the data did not show a significant change when compared with the normal lung tissue specimens $(\mathrm{p}<0.086)$. Since the gene quantity data was derived from the same set of tumors as the expression data, we also tested for a correlation between increased gene quantity and increased mRNA levels. As expected, we did not find a correlation between 14-3-3 $\gamma$ gene amplification and overexpression of 14-3-3 $\gamma$ mRNA (p value: 0.336; Pearson correlation coefficient: 0.085 ). Therefore, the elevated levels of 14-3-3 $\gamma$ mRNA could not be attributed to an increase in gene copy number. 


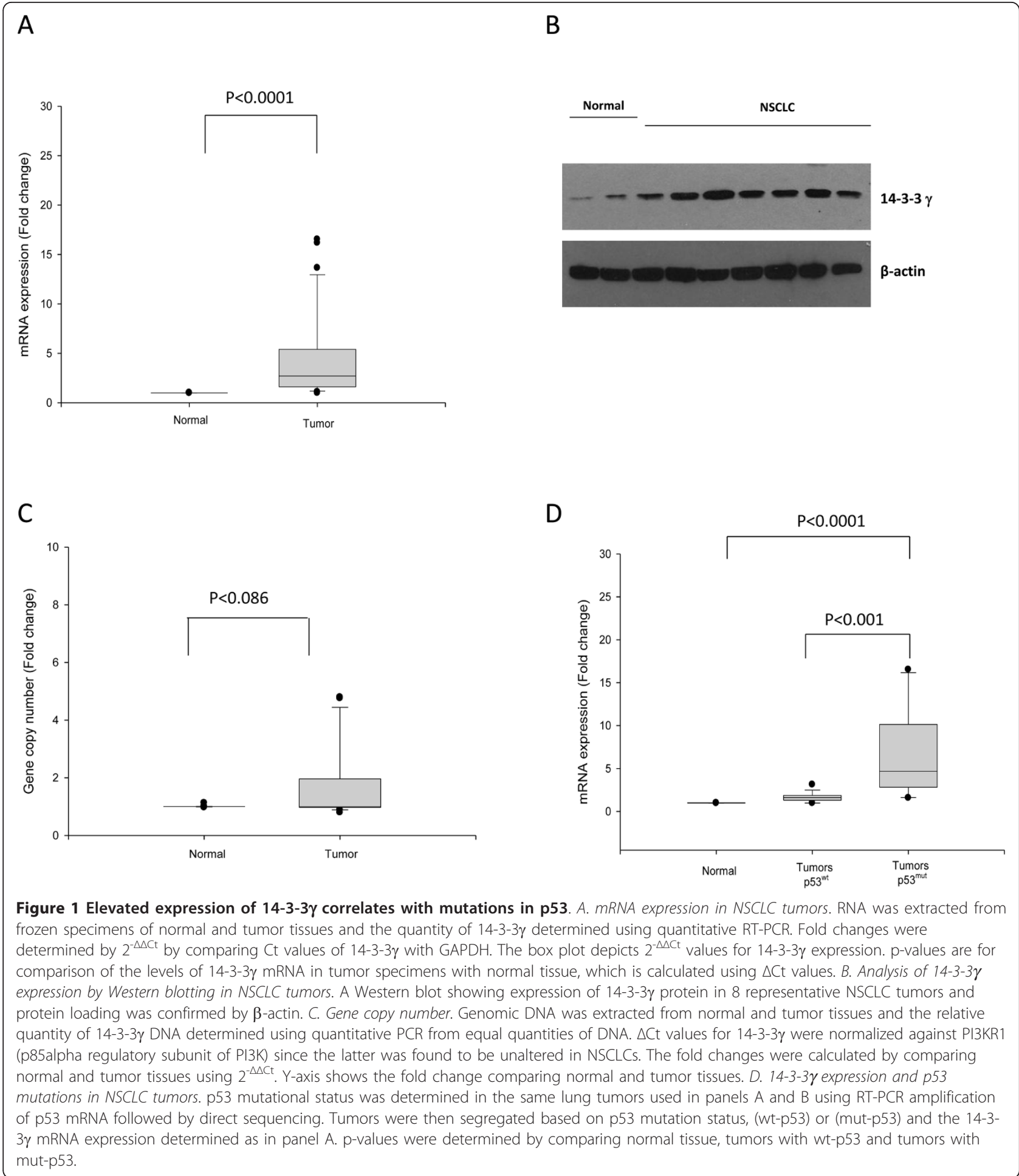

\section{Relationship between 14-3-3 $\gamma$ overexpression and p53 mutational status}

The observation by Hermeking and coworkers [3] showing that p53 and 14-3-3 are functionally related prompted us to determine whether expression of 14-3-
$3 \gamma$ was influenced by p53 mutational status. To test this, we determined the p53 mutational status in our bank of tumors as described in methods and compared this with 14-3-3 $\gamma$ mRNA expression levels. When we compared 14-3-3 $\gamma$ expression with the occurrence of 
p53 mutations (Figure 1D), we found a significant correlation between elevated 14-3-3 $\gamma$ RNA levels and mutations in p53 suggesting a functional interaction between $\mathrm{p} 53$ mutations and increased 14-3-3 $\gamma$ expression. The 14-3-3 $\gamma$ protein expression in lung tumors was also significantly correlated with p53 mutations (p $<0.0001$, Table 3$)$. We also tested for a correlation between p53 mutations and 14-3-3 $\gamma$ gene amplification. We found no significant difference when we compared 14-3-3 $\gamma$ gene amplification with p53 mutational status (data not shown). Therefore, elevated 14-3-3 $\gamma$ gene expression occurs in tumors which have mutations in p53.

\section{Repression of 14-3-3 $\gamma$ mRNA and protein expression by wt-p53}

Our observations in human lung tumors suggested that wt-p53 might suppress 14-3-3 $\gamma$ expression. To explore this further we began by testing whether activation of p53 using ionizing radiation had any effect on 14-3-3 $\gamma$. We exposed three cell lines, A549 (wt-p53), H358 (no p53 expression) and H322 (mut-p53) to ionizing radiation and then examined the levels of 14-3-3 $\gamma$ expression. As shown in Figure 2A, strong induction of p53 levels was associated with a decrease in the expression of 14$3-3 \gamma$ in response to $\gamma$-irradiation. In contrast, no induction of p53 protein was observed in either $\mathrm{H} 358$ or H322 cells. The same trend was observed in $\gamma$-irradiated HCT116 p53 $3^{+/+}$and HCT116 p53 ${ }^{-/-}$cells (data not shown), suggesting that 14-3-3 $\gamma$ expression is regulated by $\mathrm{p} 53$. To confirm that the reduced 14-3-3 $\gamma$ expression was due to p53 and not an indirect effect of ionizing radiation, we examined 14-3-3 $\gamma$ mRNA and protein levels after infection of A549 cells with a p53 expressing adenovirus. 14-3-3 $\gamma$ protein levels were consistently decreased after transduction with wild-type Adp53 but not with the GFP-adenovirus control (Figure 2B). Consistent with this, qRT-PCR showed that 14-3-3 $\gamma$ mRNA was decreased almost $30 \%$ in cells infected with the wtp53-adenovirus when compared with the GFP-adenovirus control (Figure 2C). Conversely, suppression of p53 expression in A549 cells using siRNA led to increased 14-3-3 $\gamma$ protein expression (Figure 2D). These experiments indicated that p53 could suppress the expression of $14-3-3 \gamma$.

Table 3 Expression of 14-3-3 $\gamma$ correlated with mutant p53

\begin{tabular}{llll}
\hline & $\begin{array}{l}\text { No. cancer } \\
\text { tissues }\end{array}$ & $\begin{array}{l}\text { 14-3-3 } \boldsymbol{\gamma} \text { expression } \\
\text { (> 2-fold, \%) }\end{array}$ & $P$ \\
\hline Total & 80 & $49(61.2)$ & \\
P53 wild-type & 35 & $33(67.3)$ & 0.0001 \\
P53 mutant & 45 & $16(32.7)$ & \\
\hline
\end{tabular}

Human 14-3-3 $\gamma$ gene contains a putative p53 consensus binding element

Modulation of 14-3-3 $\gamma$ expression by p53 could be either indirect, that is, by inducing another protein which in turn might regulate 14-3-3 $\gamma$ expression, or direct, by p53 itself binding to the promoter region of 14-3-3 $\gamma$. To determine whether or not p53 could bind to the promoter region of $14-3-3 \gamma$ we utilized the GFP $_{\text {UV }}$ one-hybrid system, using as "bait" 600 or 1200 bp fragments of the promoter region 5 ' to the start codon and as "prey", mouse p53. Under long wave UV light, budding yeast co-transformed with bait and prey plasmids exhibited strong green fluorescence with 1200 bp promoter fragment. Quantification of GFP emission revealed that the $1200 \mathrm{bp}$ fragment produced a significant GFP signal when compared with sucrose control which indicated that p53 could bind with the 14-3-3 $\gamma$ promoter (Figure 3A).

p53 protein is a transcription factor that specifically recognizes and binds to DNA consensus sequences defined as PuPuPuC(A/T) (T/A)GPyPyy $(\mathrm{N})_{0-14} \mathrm{PuPu}-$ $\mathrm{PuC}(\mathrm{A} / \mathrm{T})(\mathrm{T} / \mathrm{A}) \mathrm{GPyPyPy}$, in which $\mathrm{Pu}$ stands for purine, Py stands for pyrimidine, and $\mathrm{N}$ stands for any nucleotide. Analysis of the human 14-3-3 $\gamma$ promoter sequence using the TF search program revealed the presence of a putative p53 consensus binding element in the promoter (Figure 3B). To determine if p53 would indeed bind to the putative p53 consensus binding elements in human 14-3-3 $\gamma$ gene in vivo, ChIP assays were performed in A549, H322, H358, HCT116 $\mathrm{p}^{+/+}$and HCT116 p53 $3^{-/-}$ cells that were either untreated or exposed to $10 \mathrm{~Gy}$ gamma radiation. We found evidence of p53 binding to the response element in cells that expressed a wt-p53 and that this effect was enhanced with exposure to ionizing radiation. Little or no binding was observed in cells that expressed no or mutant p53 (Figures 3C \&3D).

\section{Endogenous wt-p53 inhibited the promoter activity of 14-} 3-3 $\gamma$

To confirm that p53 could suppress 14-3-3 $\gamma$ expression we cloned $1200 \mathrm{bp}$ of the 14-3-3 $\gamma$ promoter and inserted it into the pGL3 luciferase reporter plasmid and transfected this into A549, H322 or H358 cells which were either left untreated or exposed to gamma radiation. Consistent with our CHIP assays, we found that reporter activity was suppressed by as much as $\sim 50 \%$ when the cells were exposed to radiation, but that reporter activity was not affected in cells that expressed no or a mutant p53 (Figures 3E). The repression of 14-3-3 $\gamma$ promoter activity by ectopic expression of wt-p53 suggested that endogenous p53 could inhibit or modulate $14-3-3 \gamma$ promoter activity. To test whether the p53 repression of 14-3-3 $\gamma$ is a physiologically relevant response we examined the ability of endogenous p53 in A549 cells to 


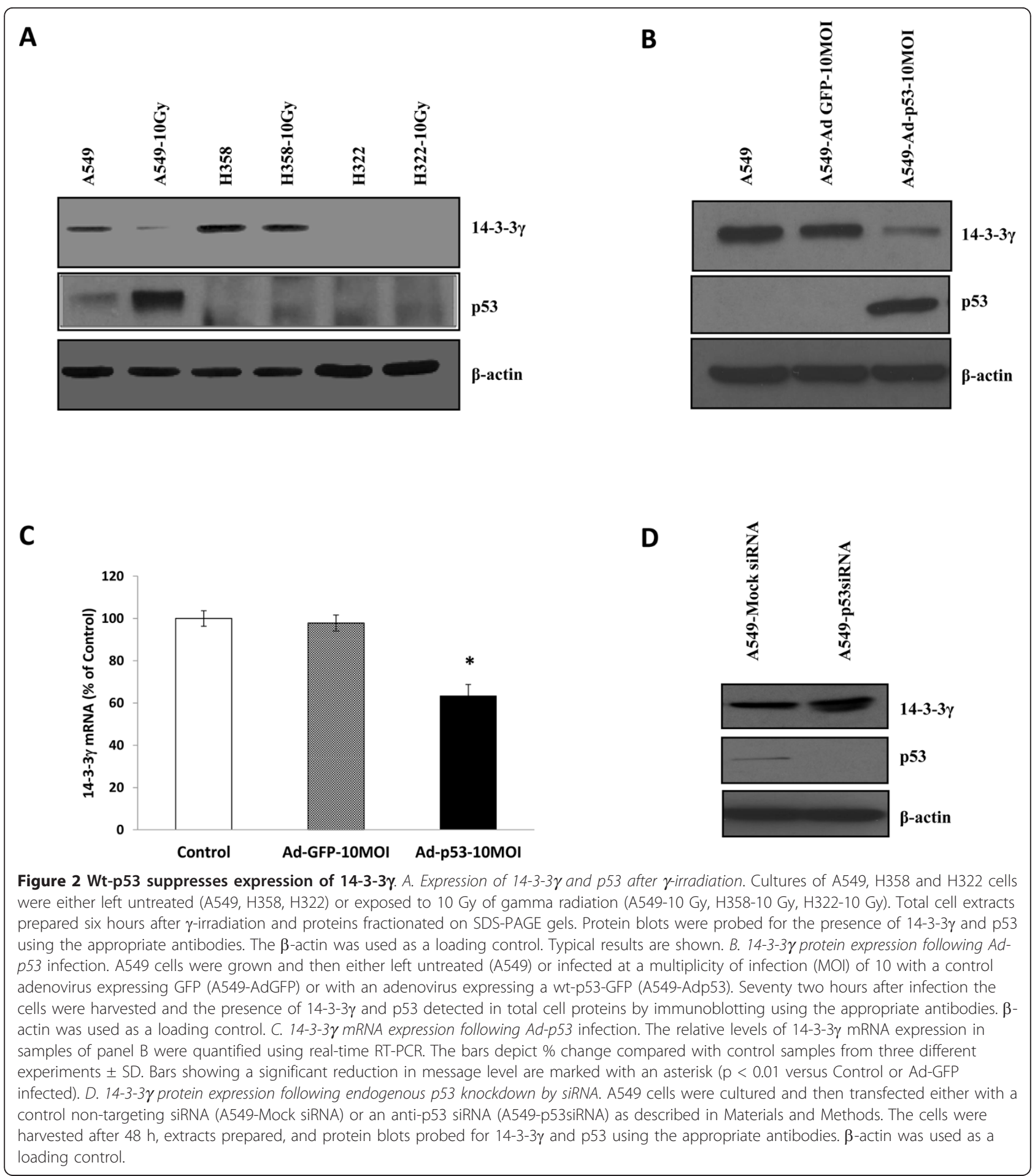

repress $14-3-3 \gamma$ promoter activity in response to gamma radiation, a known potent p53 inducer [24]. We reasoned that if $14-3-3 \gamma$ is a p53 target gene for repression, the 14-3-3 $\gamma$ promoter activity should be reduced after p53 induction in cells containing wt-p53 (Figure 3E). Indeed, we found that 14-3-3 $\gamma$ promoter activity was reduced in irradiated A549 cells, but not in either H358 or H322 cells. To further test this we also examined reporter activity in $\mathrm{HCT} 116 \mathrm{p} 53^{+/+}$and $\mathrm{HCT} 116 \mathrm{p} 53^{-/-}$ cells. Consistent with our results in A549 cells we found that reporter activity was reduced in irradiated HCT $116 \mathrm{p} 53^{+/+}$but not in HCT116p $53^{-/-}$cells or non- 
A

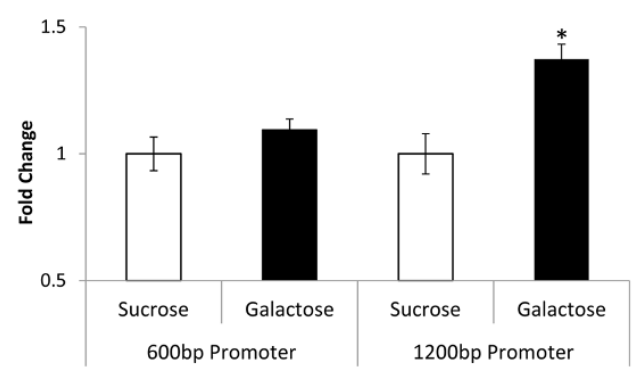

C

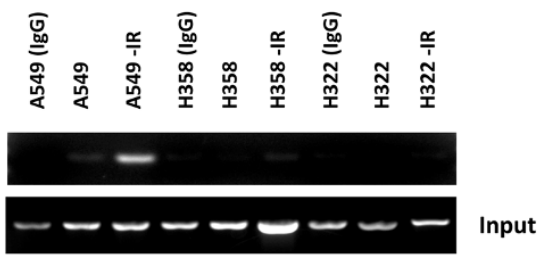

E

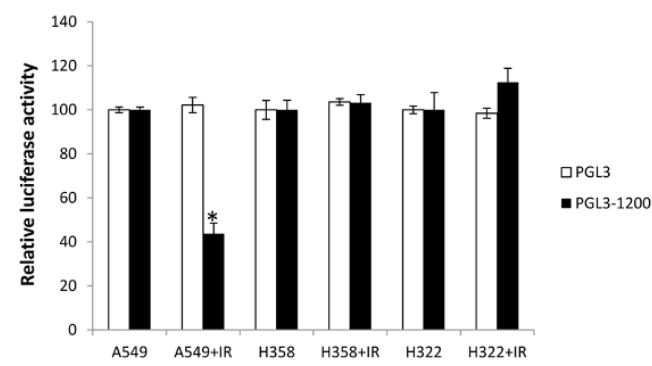

B

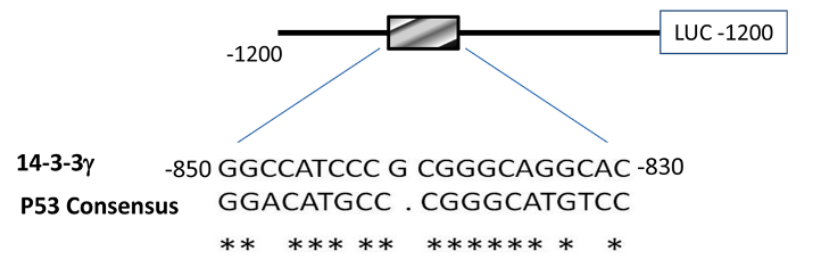

D

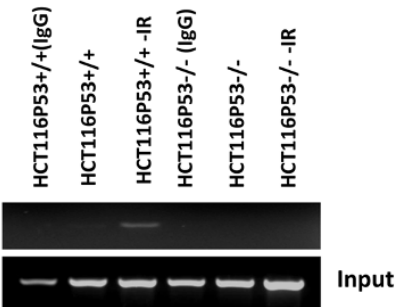

$\mathrm{F}$

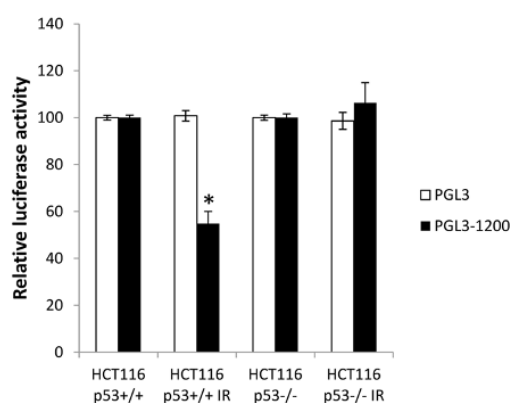

Figure 3 p53 binds 14-3-3 $\gamma$ promoter and negatively regulates 14-3-3 $\gamma$ expression. A. Yeast one hybrid assays. The 600 and 1200 bp 14-3$3 \gamma$ promoter sequences were subcloned into the GFP reporter vector as described in materials and methods. The p53 encoding plasmid was co-transformed with reporter vectors into Saccharomyces cerevisiae strain W303 and successful transformants were used for the GFP fluorescent assay in the presence of UV. The results were normalized to cells grown in the sucrose medium. Values in $y$-axis are represented as fold change. Experiments were repeated three times and $p$ values calculated using student's t-test $\left({ }^{*} p<0.05\right)$. B. A schematic diagram of the 1200 bp of the human 14-3-3 $\gamma$ gene promoter is shown with the position and sequence of the putative p53 binding motif identified. The p53 consensus binding motif is shown for comparison. Asterisks denote identity between the two sequences. This promoter fragment was cloned into PGL3 adjacent to the luciferase gene (shown at right). C. CHIP assays in lung cancer cell lines. A549, H358 and H322 cells were either left untreated (A549, H358, H322) or exposed to 10 Gy ionizing radiation (A549-IR, H358-IR, H322-IR). The cells were harvested eight hours after $\gamma$-irradiation and chromatin immunoprecipitation performed as described in the methods section. Reactions were performed either with a mouse IgG as a negative control (A549 -p53ab, H358 -p53ab, H322 -p53ab) or the anti-p53 DO1 antibody. PCR products were separated on agarose gels and visualized by staining with ethidium bromide and viewing with UV illumination. Three independent experiments were performed. Typical results are shown. D. CHIP assays in human colon cancer cell lines. HCT116p53 $3^{+/+}$or HCT116p53 $3^{-/-}$cells were either left untreated or exposed to 10 Gy ionizing radiation and chromatin immunoprecipitation reactions performed as in panel C using either a mouse lgG as a negative control (-p53ab) or with the anti-p53 DO1 antibody. PCR products were visualized as in panel C. Reactions using input DNA are shown at the bottom of the panel. Three independent experiments were performed. A typical result is shown. E. 14-3-3 $\gamma$ promoter reporter assays in lung cancer cell lines following $\gamma$ irradiation. A549, H322, or H358 cells were transiently transfected either with empty vector (open bars) or with the reporter depicted in panel B (solid bars). Forty eight hours after transfection the cells were either left untreated (A549, H358, H322) or exposed to ionizing radiation (10 Gy). The cells were harvested eight hours after gamma-irradiation and luciferase activity measured and normalized relative to renilla luciferase to control for variations in transfection efficiency. The bars represent the average of three experiments \pm SD. An asterisk denotes those values that

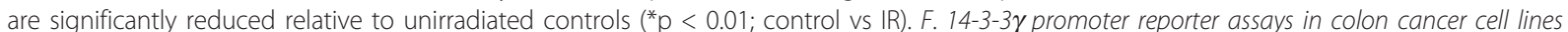

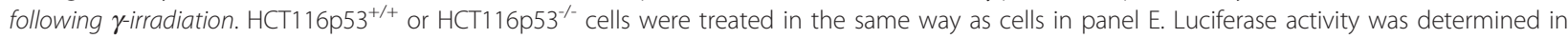
the non-irradiated and irradiated (IR) cells as described above. The bars depict the average of three independent experiments, \pm SD. Open bars show values for cells transfected with the empty vector. Solid bars show values for cells transfected with the reporter vector shown in panel B. An asterisk marks those values that were significantly reduced relative to non-irradiated controls $\left({ }^{*} p<0.01\right.$; control vs IR). 
irradiated HCT116p53 $53^{+/+}$(Figure 3F). These data strongly supported the notion that 14-3-3 $\gamma$ gene expression is negatively regulated by $\mathrm{p} 53$.

\section{The -850 to -830 region is sufficient to mediate p53 repression activity}

Finally, we constructed a set of promoter deletion mutants to test whether p53's negative regulatory activities were mediated through the putative consensus binding site. A 1200 bp stretch of DNA 5' of the 14-3$3 \gamma$ gene was cloned and deletions that removed successively larger regions from the 5' end of the sequence were generated and linked to a luciferase reporter. In addition, we constructed deletions promoter that removed either part or the entire putative p53 DNA binding motif. The deletion constructs are diagramed in Figure 4. To test reporter response to radiation activated p53, A549 cells were transiently transfected with the reporter plasmids and then either exposed to radiation or not and the luciferase activity was measured. As shown in Figure 4A, the sequences between -586 and -830 were required for activity of the $14-3-3 \gamma$ promoter and that the complete p53 consensus binding site was required for suppression of reporter activity in response to ionizing radiation.

To further test that p53 could directly repress 14-3-3 $\gamma$, we infected A549 cells with a wt-p53-GFP adenovirus or a control GFP-expressing adenovirus and measured luciferase activity (Figure 4B). As can be seen, 14-3-3 $\gamma$ promoter activity was down regulated in cells that were infected with the wt-p53 adenovirus, but not with the GFP-expressing adenovirus. Moreover, only those reporters that contained the full length p53 binding motif were negatively regulated by $\mathrm{p} 53$. To rule out the possibility that this transcriptional regulation resulted from the interference of the endogenous p53 by the p53expressing adenovirus, we also tested the promoter activity in human colon cell line HCT116p $53^{+/+}$and $\mathrm{HCT} 116 \mathrm{p} 53^{-/-}$cells. Consistent with our other experiments, activity of the 14-3-3 $\gamma$ promoter with a complete p53 response element was repressed in $\mathrm{HCT} 116 \mathrm{p} 53^{+/+}$ cells, but not in HCT116p53/- cells (Figure 4B). Taken together our results are consistent with the hypothesis that the $14-3-3 \gamma$ promoter is negatively regulated by binding to a p53 DNA binding motif in the promoter region.

\section{Discussion}

The novel finding of this study is that $14-3-3 \gamma$ is negatively regulated by $\mathrm{p} 53$ by binding to its promoter. Studies with human non small cell lung cancers have shown that expression of 14-3-3 $\gamma$ directly correlated with the p53 status, and elevated protein expression resulted from an increase in the quantity of mRNA, suggesting that there is a functional interaction between elevated 14-3-3 $\gamma$ expression and loss of p53. Although we found some evidence of 14-3-3 $\gamma$ gene amplification in some tumors, there was no significant correlation with elevated levels of gene expression. Hence, gene amplification could not account for up regulation of 143-3 $\gamma$ expression. Previously, we showed that 14-3-3 $\gamma$ caused polyploidy in lung cancer cell lines suggesting that elevated levels of expression of this family member may lead to genomic instability [19]. Therefore, it may be that increased 14-3-3 $\gamma$ expression cooperates with loss of p53 in the promotion of genomic instability in lung cancer.

Studies using in vitro experiments showed two lines of evidence suggesting that p53 repression of human 14-3$3 \gamma$ is a physiologically relevant response. First, endogenous induction of wt-p53by $\gamma$-irradiation repressed the expression of 14-3-3 $\gamma$ at the levels of mRNA and protein. Second, ectopic expression of wt-p53 significantly suppressed the expression of 14-3-3 $\gamma$. Therefore, overexpression of human wt-p53 can exert a strong inhibitory effect on human 14-3-3 $\gamma$ gene expression and tumors having mut-p53 showed strong expression. Despite the lack of studies on the regulation of 14-3-3 $\gamma$ gene expression, our findings suggest that p53 could be one of the regulators, which may, when inactivated, contribute to the elevated level of 14-3-3 $\gamma$ gene expression in tumor tissues. It is interesting to observe that wt-p53 induced repression of the human 14-3-3 $\gamma$ transcription was mediated by direct binding to its promoter. The important observation is that human 14-3-3 $\gamma$ has a p53 binding site and this site is conserved with the known reported p53-repressed genes [25]. Here the binding of p53 to its response element could result in direct repression of 14-3-3 $\gamma$ gene. Interestingly, the other 14-33 isoform, 14-3-3 $\sigma$, which is well studied, is positively regulated by p53 [3]. Even though 14-3-3 $\gamma$ protein shares more than $80 \%$ homology in protein sequence with $14-3-3 \sigma$, it is negatively regulated by $\mathrm{p} 53$. This functional difference between the two proteins is still unclear.

The finding that human $14-3-3 \gamma$ is subject to p53 repression, as reported here, provides the first linkage between p53, a powerful tumor suppressor, and 14-3-3 $\gamma$, an oncogene that promotes genomic instability and tumorigenesis. The fact that the $14-3-3 \gamma$ promoter has a p53 binding site indicates that $14-3-3 \gamma$ expression is regulated at the transcriptional level by $\mathrm{p} 53$.

\section{Conclusion}

In summary, this study has identified $14-3-3 \gamma$ as a downstream negatively regulated p53 target protein and that loss of p53 function leads to over expression of 14$3-3 \gamma$ in lung cancer. Our studies may provide the basis 


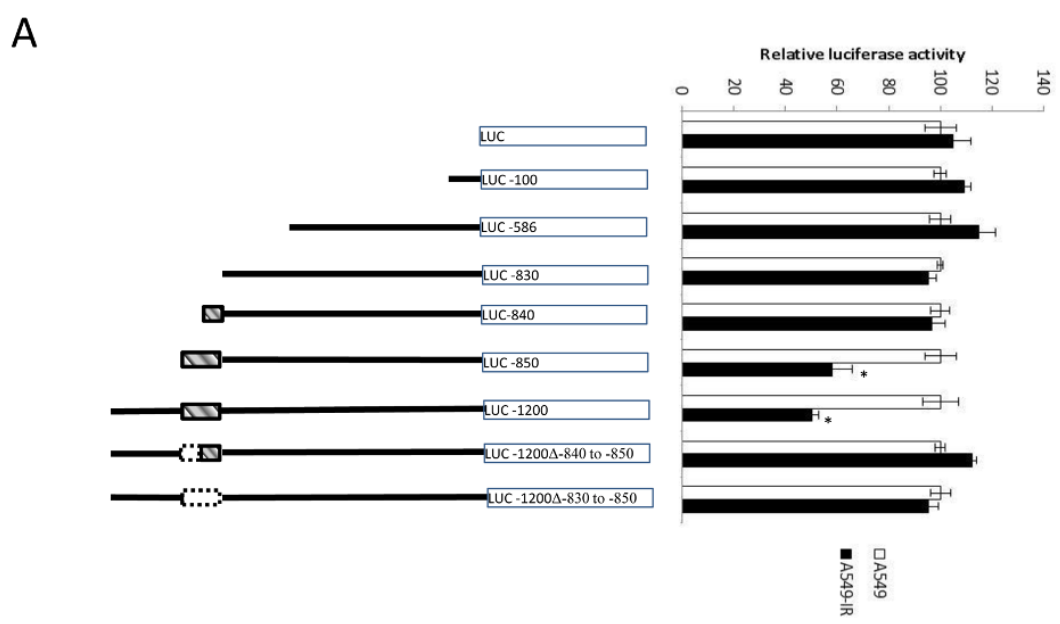

B
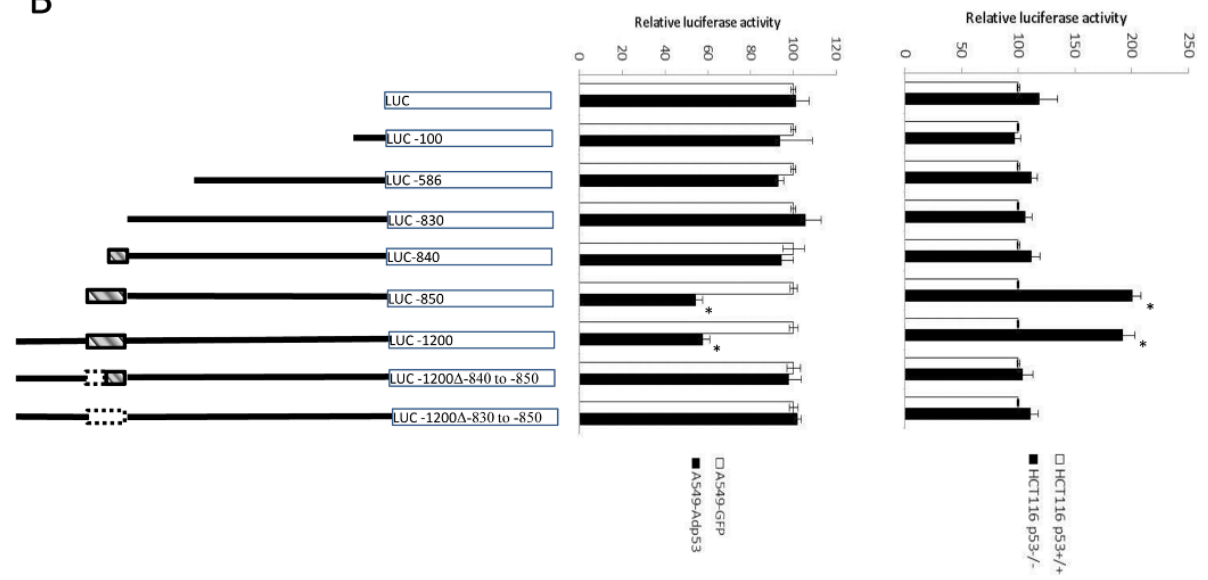

Figure 4 Deletion of the $\mathrm{p} 53$ binding motif in the 14-3-3 $\gamma$ promoter eliminates suppression by $\mathrm{p} 53$. A series of deletion mutants were constructed that successively deleted segments from the $5^{\prime}$ end of the 14-3-3 $\gamma$ promoter. These are illustrated diagrammatically linked to a luciferase gene and are shown on the left side of panels A and B. The p53 binding motif is represented as a small striped box. Small boxes outlined with stippled lines show the position of p53 consensus sequences that were deleted. The numerical designation shown within the

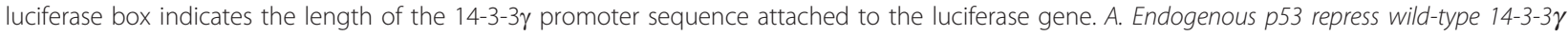

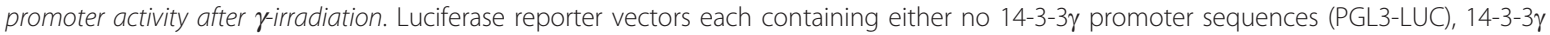
promoter sequences of various lengths (PGL3-100 bp-LUC, PGL3-586 bp-LUC, PGL3-830 bp-LUC, PGL3-1200 bp-LUC), or 14-3-3 $\gamma$ promoter sequences that lacked either all or part of the p53 consensus binding sequence (PGL3-840 bp-LUC, PGL3-850 bp-LUC, PGL3-1200 8 850-840 or PGL3-1200 $850-830$ ) were transiently transfected into A549 cells together with a renilla luciferase vector. Forty-eight hours after transfection the cells were either left untreated (open bars) or irradiated with $10 \mathrm{GY}$ ionizing radiation (solid bars). The cells were harvested 12 hours later, extracts prepared and luciferase activity quantified. The bars in the graph at the right of panel A represent the mean \pm SD of three independent experiments. Values that were determined to be significantly reduced relative to the matched control are marked with an asterisk ( $p<0.01$;

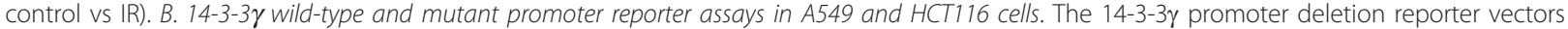
described in panel A were transfected into A549 cells that are infected with either GFP-expressing adenovirus (open bars) or with an adenovirus expressing a wt-p53 (solid bars). Forty-eight hours later the cells were harvested and luciferase activity quantified and normalized to renilla luciferase activity. Bars in the graph in the middle of panel B represent the mean of three independent experiments \pm SD. Values that were found to be significantly reduced relative to the matched control are marked with an asterisk ( $<<0.01$; Ad-GFP vs Ad-p53). In a similar experiment, $\mathrm{HCT} 116 \mathrm{p} 53^{+/+}$(open bars) and HCT116p53 (closed bars) with the same vectors described above and irradiated with $10 \mathrm{~Gy}$ ionizing radiation. Luciferase activity was determined and normalized to renilla luciferase and the values graphed. Results shown represent the mean of three independent experiments \pm SD. Values determined to be significantly reduced relative to their matched controls are marked with an asterisk ( $\mathrm{p}<0.01 ; \mathrm{HCT} 116 \mathrm{p} 53^{+/+}$vs HCT116 $\left.\mathrm{p} 53^{-1 /}\right)$. 
for further understanding of the role of 14-3-3 $\gamma$ in lung tumorigenesis and may open up potential targets for therapeutic approaches.

\section{Acknowledgements}

These studies were supported by a grant to JDM from the NIH (2R56CA107510) and a Cancer Center Support Grant to the Arizona Cancer Center (p30 CA023074).

\section{Author details}

${ }^{1}$ Arizona Cancer Center, Department of Cellular \& Molecular Medicine, University of Arizona, Tucson, Arizona 85724, USA. ${ }^{2}$ Department of Pediatrics, Steele Research Center, 1501 N Campbell Ave, Tucson, Arizona 85724, USA. 3Department of Surgery, 1501 N Campbell ave, Tucson, Arizona 85724, USA ${ }^{4}$ Department of Medicine, 1515 N. Campbell Ave., Tucson, Arizona 85724, USA

\section{Authors' contributions}

VMR carried out experimental design, molecular cloning, CHIP assay, Western blot and GPCR. CWP performed yeast hybrid assay. WQ carried out western blot for 14-3-3ץ. JDM designed the experiments and analyzed the data. VMR and JDM wrote the manuscript. All the authors have read and approved the final manuscript.

\section{Competing interests}

The authors declare that they have no competing interests.

Received: 14 March 2011 Accepted: 25 August 2011

Published: 25 August 2011

\section{References}

1. Muslin AJ, Tanner JW, Allen PM, Shaw AS: Interaction of 14-3-3 with signaling proteins is mediated by the recognition of phosphoserine. Cell 1996, 84(6):889-97.

2. Fu H, Subramanian RR, Masters SC: $14-3-3$ proteins: structure, function, and regulation. Annual review of pharmacology and toxicology 2000, 40:617-47.

3. Hermeking $H$, Lengauer $C$, Polyak $K$, et al: 14-3-3 sigma is a p53-regulated inhibitor of G2/M progression. Molecular cell 1997, 1(1):3-11.

4. Porter GW, Khuri FR, Fu H: Dynamic 14-3-3/client protein interactions integrate survival and apoptotic pathways. Seminars in cancer biology 2006, 16(3):193-202

5. Thorson JA, Yu LW, Hsu AL, et al: 14-3-3 proteins are required for maintenance of Raf-1 phosphorylation and kinase activity. Molecular and cellular biology 1998, 18(9):5229-38.

6. Ichimura T, Isobe T, Okuyama T, Yamauchi T, Fujisawa H: Brain 14-3-3 protein is an activator protein that activates tryptophan 5monooxygenase and tyrosine 3-monooxygenase in the presence of $\mathrm{Ca} 2$ +, calmodulin-dependent protein kinase II. FEBS letters 1987, 219(1):79-82.

7. Berg D, Holzmann C, Riess O: $14-3-3$ proteins in the nervous system. Nature reviews 2003, 4(9):752-62.

8. Qi W, Liu X, Qiao D, Martinez JD: Isoform-specific expression of 14-3-3 proteins in human lung cancer tissues. International journal of cancer 2005, 113(3):359-63.

9. Cheng L, Pan CX, Zhang JT, et al: Loss of 14-3-3sigma in prostate cancer and its precursors. Clin Cancer Res 2004, 10(9):3064-8.

10. Umbricht CB, Evron E, Gabrielson E, Ferguson A, Marks J, Sukumar S Hypermethylation of 14-3-3 sigma (stratifin) is an early event in breast cancer. Oncogene 2001, 20(26):3348-53.

11. Matta A, Bahadur S, Duggal R, Gupta SD, Ralhan R: Over-expression of 143-3zeta is an early event in oral cancer. BMC cancer 2007, 7:169.

12. Akahira J, Sugihashi $Y$, Suzuki T, et al: Decreased expression of 14-3-3 sigma is associated with advanced disease in human epithelial ovarian cancer: its correlation with aberrant DNA methylation. Clin Cancer Res 2004, 10(8):2687-93.

13. Guweidhi A, Kleeff J, Giese N, et al: Enhanced expression of 14-3-3sigma in pancreatic cancer and its role in cell cycle regulation and apoptosis. Carcinogenesis 2004, 25(9):1575-85.
14. Okada T, Masuda N, Fukai $Y$, et al: Immunohistochemical expression of 143-3 sigma protein in intraductal papillary-mucinous tumor and invasive ductal carcinoma of the pancreas. Anticancer research 2006, 26(4B):3105-10.

15. Chan TA, Hermeking $H$, Lengauer C, Kinzler KW, Vogelstein B: 14-3-3Sigma is required to prevent mitotic catastrophe after DNA damage. Nature 1999, 401(6753):616-20.

16. Urano T, Saito T, Tsukui T, et al: Efp targets 14-3-3 sigma for proteolysis and promotes breast tumour growth. Nature 2002, 417(6891):871-5.

17. Dellambra E, Golisano O, Bondanza S, et al: Downregulation of 14-33sigma prevents clonal evolution and leads to immortalization of primary human keratinocytes. The Journal of cell biology 2000, 149(5):1117-30.

18. Jin YH, Kim YJ, Kim DW, et al: Sirt2 interacts with 14-3-3 beta/gamma and down-regulates the activity of $\mathrm{p} 53$. Biochemical and biophysical research communications 2008, 368(3):690-5.

19. Qi W, Liu X, Chen W, Li Q, Martinez JD: Overexpression of 14-3-3gamma causes polyploidization in H322 lung cancer cells. Molecular carcinogenesis 2007, 46(10):847-56.

20. Carney DN: Oncogenes and genetic abnormalities in lung cancer. Chest 1989, 96(1 Suppl):25S-7S

21. Massion PP, Kuo WL, Stokoe D, et al: Genomic copy number analysis of non-small cell lung cancer using array comparative genomic hybridization: implications of the phosphatidylinositol 3-kinase pathway. Cancer research 2002, 62(13):3636-40.

22. Kuo $M H$, Allis CD: In vivo cross-linking and immunoprecipitation for studying dynamic Protein:DNA associations in a chromatin environment. Methods (San Diego, Calif 1999, 19(3):425-33.

23. Radhakrishnan VM, Martinez JD: 14-3-3gamma induces oncogenic transformation by stimulating MAP kinase and PI3K signaling. PloS one 5(7):e11433.

24. Fu L, Benchimol S: Participation of the human p53 3'UTR in translational repression and activation following gamma-irradiation. The EMBO journal 1997, 16(13):4117-25

25. Riley T, Sontag E, Chen P, Levine A: Transcriptional control of human p53regulated genes. Nat Rev Mol Cell Biol 2008, 9(5):402-12.

\section{Pre-publication history}

The pre-publication history for this paper can be accessed here: http://www.biomedcentral.com/1471-2407/11/378/prepub

\section{doi:10.1186/1471-2407-11-378}

Cite this article as: Radhakrishnan et al.: P53 suppresses expression of the 14-3-3gamma oncogene. BMC Cancer 2011 11:378.

\section{Submit your next manuscript to BioMed Central and take full advantage of:}

- Convenient online submission

- Thorough peer review

- No space constraints or color figure charges

- Immediate publication on acceptance

- Inclusion in PubMed, CAS, Scopus and Google Scholar

- Research which is freely available for redistribution
C Biomed Central 